\title{
Are glucagon-like peptide 1 analogues effective and safe in severe COVID-19 patients with type 2 diabetes? - a case report
}

\author{
Shuqian Chen ${ }^{1}$, Wei Lin ${ }^{1}$, Junping Wen ${ }^{1}$, Baosong Xie ${ }^{2}$, Lizhou Chen ${ }^{3}$, Gang Chen ${ }^{1}$ \\ ${ }^{1}$ Department of Endocrinology, Shengli Clinical Medical College of Fujian Medical University, Fujian Provincial Hospital, Fuzhou, China; \\ ${ }^{2}$ Department of Respiratory, Shengli Clinical Medical College of Fujian Medical University, Fujian Provincial Hospital, Fuzhou, China; ${ }^{3}$ Department \\ of Respiratory, Fuzhou Pulmonary Hospital of Fujian Province, Fuzhou, China \\ Correspondence to: Baosong Xie, MD. Department of Respiratory, Shengli Clinical Medical College of Fujian Medical University, Fujian Provincial \\ Hospital, Fuzhou, China. Email: xbaosong@126.com; Lizhou Chen, MD. Department of Respiratory, Fuzhou Pulmonary Hospital of Fujian \\ Province, Fuzhou, China. Email: chenlizhou199@163.com; Gang Chen, MD. Department of Endocrinology, Shengli Clinical Medical College of \\ Fujian Medical University, Fujian Provincial Hospital, Fuzhou, China. Email: chengangfj@163.com.
}

\begin{abstract}
Many cases of novel coronavirus 2019 (COVID-19) have confirmed in many countries around the world. Due to the disorders of the immune system, diabetic patients are more likely to suffer from severe COVID-19. Glucagon-like peptide 1 analogues (GLP-1 analogues) commonly can be used to reduce blood sugar. There is no clear evidence that it can be safely and effectively used in patients with diabetes merged severe COVID-19. In this case, we described A 65-year-old male with hypertension and diabetes was diagnosed with severe COVID-19, he took liraglutide at doses ranging from 0.8 to $1.8 \mathrm{mg}$. Before admission, liraglutide was not used to reduce blood glucose. Hydroxychloroquine sulfate and abidol were used to antivirus and supportive treatment were used simultaneously during hospitalization. During treatment, the patient's own state was paid attention to, and blood glucose, liver function, kidney function, white blood cells, lymphocytes and other indicators were checked and chest CT was reviewed regularly, which could reflect changes in disease. After treatment, the patient's blood glucose was under control, and his liver function, renal function, white blood cells, lymphocytes and other indicators were normal and chest CT also improved. The case showed that liraglutide may be effective and safe used in patients with severe COVID-19 combined with type 2 diabetes, but more clinical trials are needed.
\end{abstract}

Keywords: Case report; liraglutide; severe novel coronavirus 2019 (severe COVID-19); type 2 diabetes

Submitted Oct 06, 2020. Accepted for publication Nov 20, 2020.

doi: 10.21037/apm-20-1982

View this article at: http://dx.doi.org/10.21037/apm-20-1982

\section{Introduction}

Diabetes patients are more susceptible to severe novel coronavirus 2019 (COVID-19) because of the multiple disorders of the immunization system (1). GLP-1 analogues can have an anti-inflammatory effect in the human body. However, there is not enough evidence to show that GLP-1 analogues can be safely used in severe patients with coronavirus infection (2). This paper introduces a case of severe COVID-19 patient combined with diabetes. As we know, GLP-1 analogues can be used in COVID-19 patients combined with diabetes (3).

We present the following article in accordance with the CARE reporting checklist (available at http://dx.doi. org/10.21037/apm-20-1982).

\section{Case presentation}

A 65-year-old male with hypertension, diabetes was admitted to hospital. He didn't have dehydration. His nucleic acid test was positive and was diagnosed with severe COVID-19 (at admission, oxygen saturation was less than $93 \%$ in the resting state). He neglected to treat his diabetes and his $\mathrm{HbAlc}$ was $9.7 \%(83 \mathrm{mmol} / \mathrm{mol})$ at admission. He took anti-hyperglycemia drugs irregularly. The specific 


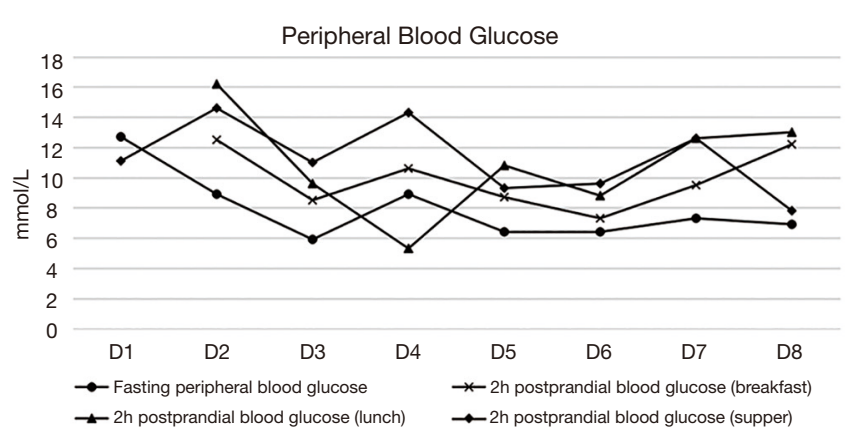

Figure 1 The patient had good blood glucose control. D, day; h, hour.

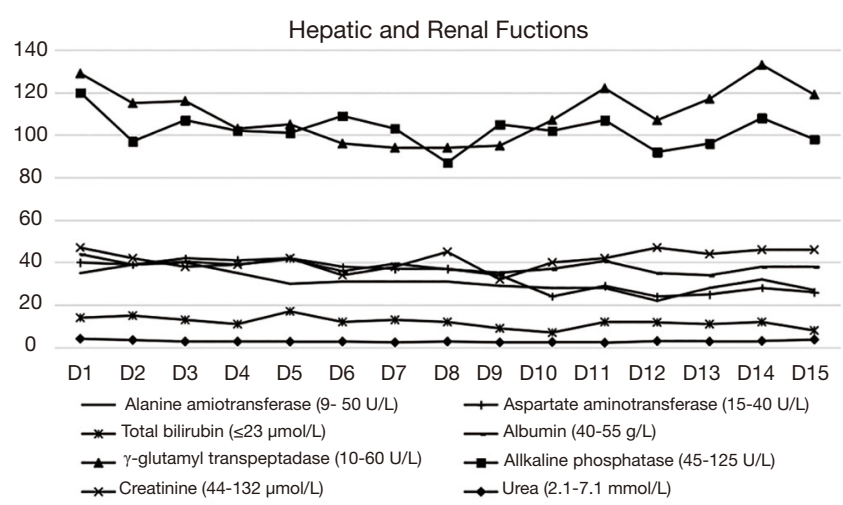

Figure 2 The hepatic and kidney functions were good during the treatment. D, day.

oral medications were not available. The patient had not been injected with liraglutide before; $0.6 \mathrm{mg}$ of liraglutide was subcutaneous injected once a day to reduce blood glucose after admission, and then gradually increased to $1.8 \mathrm{mg}$. Lantus can be added if the patient's blood glucose was above goal, Dynamic change of blood glucose level was shown in Figure 1. Hydroxychloroquine sulfate and abidol were used to antivirus and supportive treatment were used simultaneously during hospitalization. Dynamic change of liver function, renal function, white blood cells, lymphocytes and inflammatory indicators during treatment were shown in Figures 2 and 3. The patient's condition improved gradually, blood glucose was under control, the laboratory test and chest CT also improved after treatment (Figure 4).

All procedures performed in studies involving human participants were in accordance with the ethical standards of the institutional and/or national research committee(s) and with the Helsinki Declaration (as revised in 2013). Written informed consent was obtained from the patient. This study

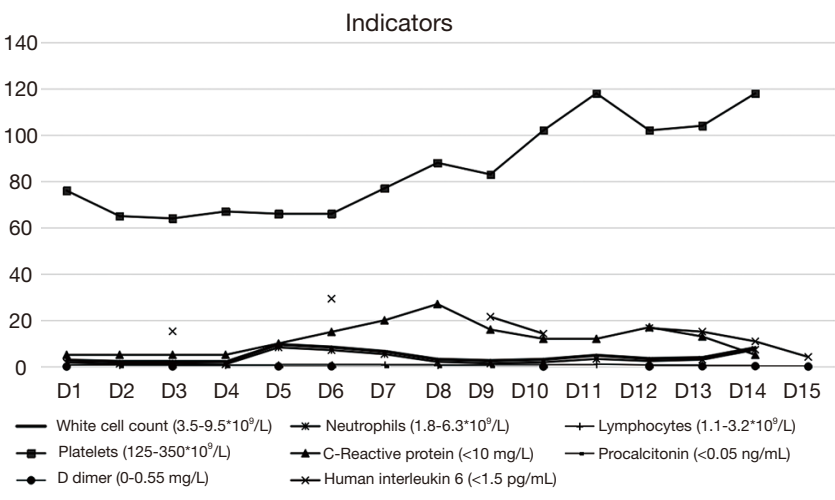

Figure 3 During the treatment, the patient's inflammatory markers and other indicators gradually returned to normal. D, day.

was approved by the Ethics Committee of Fujian Provincial Hospital (Batch number: K2020-03-001).

\section{Discussion}

Advanced age, diabetes and hypertension increase the severity and mortality in patients with COVID-19. The severity of diabetes was positively correlated with poor prognosis (4). Diabetic patients have immune dysfunction. Patients with diabetes and severe COVID-19 have higher proinflammatory cytokines than non-diabetics (1). There are at least two reasons why hyperglycemia can be very dangerous during the SARS-CoV-2 infection. The first is the blood sugar rise sharply with the tremendous increase of the inflammatory medium, another reason is the binding of SARS-CoV-2 to ACE2 (5). High blood glucose promotes the synthesis of advanced glycation end products (AGEs) and pro-inflammatory cytokines, oxidative stress, and the production of adhesion molecules that stimulate tissue inflammation abnormal immune cell response and immune abnormalities are thought to play a significant role in the severity of virus-induced diseases (6). SARS-CoV2 may cause diabetes by binding to ACE2 present in islet cells. ACE2 is highly expressed in islet cells of alveoli, pancreas, heart and kidney. Increased expression of ACE2 was found in lung tissue of subjects with diabetes. Hyperglycemia changes the binding of virus spike protein to ACE2 and immune response to virus through the potential changes of glycosylation of ACE2 and glycosylation of virus spike protein. In uncontrolled hyperglycemia, potential hyperglycosylated ACE2 in lungs, nasal airways, tongue and oropharynx may also increase SARS-CoV2 virus binding sites, resulting in higher COVID-19 tendency (7). 

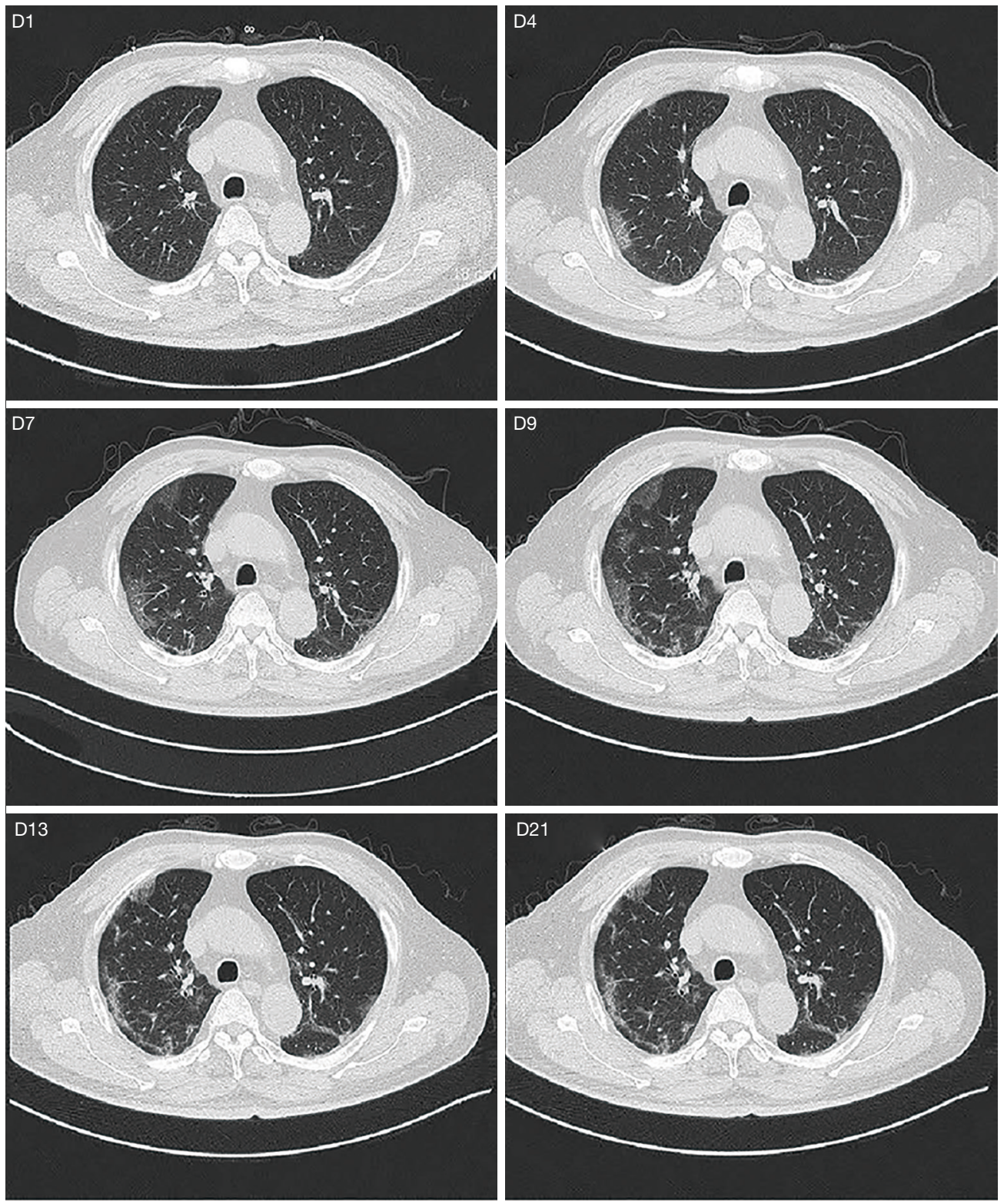

Figure 4 Chest CT showed lung lesions of day 4 were more GGO lesions compared with day 1, some old lesions dissipated or developed into consolidation on day 7, GGO lesions emerged on day 9, lung lesions were more stable on day 13 and most of the lesions disappeared on day 21, leaving slight remnant consolidation. GGO, ground-glass opacity; D, day.

However, it is well known that hyperglycemia up-regulates the expression of ACE2, making the cells vulnerable to the inflammatory and damaging effect of the virus (3). As a safe hypoglycemic drug, GLP-1 analogues have been shown to have other beneficial effects in addition to hypoglycemic effects, such as anti-inflammatory effects. In a rat model of severe endotoxemia, liraglutide reduced mortality, suppressed inflammation, reduced oxidative stress, suppressed inflammatory mediator production, prevented thrombocytopenia and microvascular thrombosis in pulmonary vasculature (8). GLP-1 analogues such as liraglutide show anti-inflammatory activity by activating adenylate cyclase (AC) to produce cyclic adenosine monophosphate (CAMP), then activating protein kinase 
A (PKA) to activate CAMP response element binding protein (CREB). Elevated IL-6 level is an independent predictor of type 2 diabetes, and studies have shown that liraglutide could reduce IL-6 level (9). Good control of blood glucose can significantly reduce overall adverse outcomes and mortality. Safe and effective control of blood sugar is the focus of work, but hypoglycemia is a negative prognostic factor for critically ill patients. Studies have shown that GLP-1 is seldom associated with hypoglycemia. But what we should know is that IL-6 may be a marker instead of maker of disease. Also, IL-6 is not specific for diabetes. In this case, the patient's blood glucose was well controlled by liraglutide, his liver function and renal function were normal and his IL-6 level became normal on day 15. Our experience showed that liraglutide could be safely used in severe COVID-19 patients and may play a role in reducing IL-6 level. This hypothesis needs clinical trials to confirm.

There is another important point, but it is easy to overlook, that is the safety of health care personnel. During an epidemic, the intensive work of nurses may lead to a decrease in resistance, so it is important to reduce the workload and exposure time of nurses. Compared with intensive insulin therapy, anti-hyperglycemia therapy with daily injection of such as liraglutide reduces the frequency of contact between nurses and COVID-19 patients, thus it can reduce the risk of transmission from patients to nurses (10). Good control of blood glucose and reducing the frequency of hypoglycemia during inpatient periods can reduce the overwork of health care workers by reducing fingerstick testing, patient discomfort.

\section{Conclusions}

In this case, the improvement of the patient's condition may in part because of good blood glucose control by liraglutide. The patient's liver function, renal function and other indicators were normal during treatment, which indicated that liraglutide can be safely used in patients with severe COVID-19 combined with type 2 diabetes. The limitation of this article is that it contains one specific case only, more clinical trials may be needed to confirm this hypothesis. But it can still be considered a valuable experience and supply some insights how to treat severe COVID-19 patients with type 2 diabetes.

\section{Acknowledgments}

We greatly appreciate the efforts of all the hospital employees and their families at the Fujian Provincial Hospital, Fuzhou Pulmonary Hospital of Fujian Province and affiliated Hospital of Fujian Medical University, who are working tirelessly during this outbreak.

Funding: None.

\section{Footnote}

Reporting Checklist: The authors have completed the CARE reporting checklist. Available at http://dx.doi.org/10.21037/ apm-20-1982

Conflicts of Interest: All authors have completed the ICMJE uniform disclosure form (available at http://dx.doi. org/10.21037/apm-20-1982). The authors have no conflicts of interest to declare.

Ethical Statement: The authors are accountable for all aspects of the work in ensuring that questions related to the accuracy or integrity of any part of the work are appropriately investigated and resolved. All procedures performed in studies involving human participants were in accordance with the ethical standards of the institutional and/or national research committee(s) and with the Helsinki Declaration (as revised in 2013). Written informed consent was obtained from the patient. This study was approved by the Ethics Committee of Fujian Provincial Hospital (Batch number: K2020-03-001).

Open Access Statement: This is an Open Access article distributed in accordance with the Creative Commons Attribution-NonCommercial-NoDerivs 4.0 International License (CC BY-NC-ND 4.0), which permits the noncommercial replication and distribution of the article with the strict proviso that no changes or edits are made and the original work is properly cited (including links to both the formal publication through the relevant DOI and the license). See: https://creativecommons.org/licenses/by-nc$\mathrm{nd} / 4.0 /$.

\section{References}

1. Shenoy A, Ismaily M, Bajaj M. Diabetes and covid-19: a global health challenge. BMJ Open Diabetes Res Care 2020;8:e01450.

2. Drucker DJ. Coronavirus Infections and Type 2 DiabetesShared Pathways with Therapeutic Implications. Endocr Rev 2020;41:bnaa011. 
3. Bornstein SR, Rubino F, Khunti K, et al. Practical recommendations for the management of diabetes in patients with COVID-19. Lancet Diabetes Endocrinol 2020;8:546-50.

4. Hill MA, Mantzoros C, Sowers JR. Commentary: COVID-19 in patients with diabetes. Metabolism 2020;107:154217.

5. Bloomgarden $\mathrm{Z}$. Is the type of diabetes treatment relevant to outcome of COVID-19? J Diabetes 2020;12:486-7.

6. Liu Y, Du X, Chen J, et al. Neutrophil-to-lymphocyte ratio as an independent risk factor for mortality in hospitalized patients with COVID-19. J Infect 2020;81: e6-e12.

7. Sharma S, Ray A, Sadasivam B. Metformin in COVID-19:
A possible role beyond diabetes. Diabetes Res Clin Pract 2020;164:108183.

8. Shah FA, Mahmud H, Gallego-Martin T, et al. Therapeutic Effects of Endogenous Incretin Hormones and Exogenous Incretin-Based Medications in Sepsis. J Clin Endocrinol Metab 2019;104:5274-84.

9. Brock C, Hansen CS, Karmisholt J, et al. Liraglutide treatment reduced interleukin-6 in adults with type 1 diabetes but did not improve established autonomic or polyneuropathy. Br J Clin Pharmacol 2019;85:2512-23.

10. Huang L, Lin G, Tang L, et al. Special attention to nurses' protection during the COVID-19 epidemic. Crit Care 2020;24:120.

Cite this article as: Chen S, Lin W, Wen J, Xie B, Chen L, Chen G. Are glucagon-like peptide 1 analogues effective and safe in severe COVID-19 patients with type 2 diabetes? - a case report. Ann Palliat Med 2021;10(6):7057-7061. doi: 10.21037/ apm-20-1982 